\title{
Establishing Best Practices for Photogrammetric Recording During Archaeological Fieldwork
}

\author{
Philip Sapirstein and Sarah Murray \\ University of Nebraska-Lincoln, Lincoln, Nebraska
}

\begin{abstract}
Archaeologists have recently embraced photogrammetry as a low-cost, efficient tool for recording archaeological artifacts, active excavation contexts, and architectural remains. However, no consensus has yet been reached about standard procedures for reliable and metrically accurate photogrammetric recording. The archaeological literature describes diverse equipment and approaches to photogrammetry. The purpose of this article is to open a discussion about when and how photogrammetry should be employed in archaeology in an effort to establish "best practices" for this new method. We focus on the integration of photogrammetry within a comprehensible research plan, the selection of equipment, the appropriate apportionment of labor and time on site, and a rubric for site photography that is conducive to successful and efficient modeling. We conclude that photogrammetric modeling will soon become an indispensable tool in most archaeological applications but should always be implemented in ways that do not place undue burdens on project personnel and budgets and that aid research goals in well-defined ways.
\end{abstract}

\section{KEYWORDS}

Photogrammetry; research design; accuracy; efficiency; best practices

\section{Introduction}

These days photogrammetry is all the rage. Many archaeological projects are shifting to fully digital, three-dimensional (3D) recording systems based on the fast, efficient, and inexpensive modeling made possible by new software. The Tel Akko Total Archaeology project has relied on photogrammetric recording for excavation since 2012 (Olson et al. 2013), as have many other projects (De Reu et al. 2014; Levy et al. 2014; Douglass et al. 2015; Opitz et al. 2016; Roosevelt et al. 2015; Sapirstein 2015, 2016).

While it is currently experiencing a boom, the adoption of 3D technology at archaeological sites is not a new development. A sophisticated understanding of 3D space has always been a prerequisite for the recording and interpretation of spatially complex archaeological data. Archaeologists have consistently been early adopters of 3D recording technology, such as laser scanning. However, costs have historically been prohibitive for many projects. In contrast, packages for automated photogrammetry released in the past several years allow users to create 3D models within a smaller budget, making $3 \mathrm{D}$ recording much more accessible.

The latest generation of photogrammetry software is characterized by the replacement of earlier "close-range" methods-designed for individual point measurement and relying on coded survey targets to estimate relative locations of cameras within a scene (Luhmann 2010) - with highly automated alternatives. The latter, often labeled "imagebased modeling," first determines camera locations by methods known as Structure from Motion (SfM), and then computes dense 3D surface models of the scene with MultiView Stereo (MVS or DMVR) (Koutsoudis et al. 2013). Here, we use "photogrammetry" to refer generally to the procedures of image-based modeling and distinguish the SfM and MVS stages when appropriate.
Photogrammetric recording has many benefits, especially in efficiency and detail (Koutsoudis et al. 2014; Lerma and Muir 2014; Douglass et al. 2015; Sapirstein 2015). Nonetheless, its adoption is not without concomitant drawbacks. A site that integrates photogrammetry must assign personnel to preparing the subjects, photography, and computer processing. The data produced are wholly digital and thus demand infrastructure for archiving photographs, working files, 3D models, and rendered illustrations. As a result, relatively few projects have released much of their digital data (but, recently, see Opitz et al. 2016). Another concern with any $3 \mathrm{D}$ recording system is the opacity of the technology. When digitizing pottery with a structured-light scanner or modeling terrain from aerial photographs taken by an Unmanned Aerial Vehicle (UAV), archaeologists surrender archaeological documentation to technologies whose underlying operations are so complex that they become impossible for the human user to fully understand (Rabinowitz 2015).

Nonetheless, 3D recording is innately valuable to archaeological research, and we believe the field is moving in the right direction by turning increasingly to digital methods for recording and analysis. Here, we argue that it is necessary to move beyond the initial phase of wonderment at our new capabilities to create life-like 3D imagery from a set of photographs to the matter of creating a consensus about what should constitute standard practice for archaeological photogrammetry. Our focus is on data collection during fieldwork, in particular the large-scale recording of trenches and architecture, rather than on small-object photography-a process deserving consideration on its own right (Koutsoudis et al. 2013; Porter et al. 2016). Steps for processing photogrammetric models are also too complex to address effectively here, and we instead refer the reader to previous discussions (Olson et al. 2013; Douglass et al. 2015). 